\title{
Cáncer oral: Genética, prevención, diagnóstico y tratamiento. Revisión de la literatura
}

\section{Oral cancer: Genetics, prevention, diagnosis and treatment. Literature review}

\author{
Mateo-Sidrón Antón MC*, Somacarrera Pérez ML**
}

\section{RESUMEN}

El objetivo de esta revisión es actualizar la situación actual del cáncer oral, así como de otros aspectos en general de esta enfermedad y del mecanismo genético y tumorogénesis del cáncer. Para ello hemos revisado los trabajos publicados de los últimos diez años sobre todos los aspectos generales del cáncer oral, destacando, entre otros, los referentes a factores de riesgo, diagnóstico y tratamiento. Las conclusiones más importantes de esta revisión han sido que, el cáncer oral, es un problema que aumenta con la edad, a partir de los 50 años en adelante, y que el $90 \%$ de los tumores que aparecen en boca son carcinomas mucoepidermoides de células escamosas. Es una enfermedad multifactorial, pero dentro de los factores de riesgo, se destacan el papel del tabaco y el alcohol. La prevalencia del cáncer oral sigue en aumento, por lo que el papel del odontólogo es primordial para la detección precoz de estos procesos, realizando una correcta historia clínica y exploración extra e intraoral, consiguiendo disminuir su morbimortalidad.

Palabras clave: Cáncer oral, ciclo celular, oncogenes, proteína P53, tabaco, leucoplasia, eritroplasia, metástasis, radioterapia, quimioterapia.

\section{SUMMARY}

The aim of this revision was to update the actual situation of oral cancer, and other important aspects of this illness explaining how the genetic mechanism and tumorogenesis in cancer works. We have reviewed the articles published in the last ten years about the general aspects of oral cancer, distinguishing the risk factors, diagnosis and treatment. The most important conclusions of this review, in relation to oral cancer, have been that this illness is a problem which increases with age, from 50 years old onwards, and that around $90 \%$ of the tumours that appear in the oral cavity are squamous cell carcinomas. Oral cancer is multifactorial, but the two most important risk factors are tobacco and alcohol. The prevalence of oral cancer continues to rise, that is why the role of the dentist is very important in detecting this illness in its early stages, by doing a correct anamnesis and a correct extraoral and intraoral inspection, decreasing the morbility and mortality.

Key words: Oral cancer, cell cycle, oncogenes, protein P53, tobacco, leukoplakia, eritroplakia, metastases, radiotherapy, chemothera.

Fecha de recepción: 2 de octubre de 2014.

Aceptado para publicación: 15 de enero de 2015.

* Licenciada en odontología, Universidad Europea de Madrid. Especialista en Medicina Oral, Universidad Rey Juan Carlos.

** Catedrática de Medicina Oral. Universidad Europea de Madrid..

Mateo-Sidrón Antón MC, Somacarrera Pérez ML Cáncer oral: Genética, prevención, diagnóstico y tratamiento. Revisión de la literatura. Av. Odontoestomatol 2015; 31 (4): 247-259. 


\section{INTRODUCCIÓN}

La OMS; define el término cáncer como un amplio grupo de enfermedades que pueden afectar a cualquier parte del cuerpo (1). El cáncer supone un problema de salud a nivel mundial y es una de las causas más importantes de mortalidad $(2,3)$. Las células cancerosas proliferan sin medida, ajenas al control de los organismos pluricelulares, por medio de mitosis repetidas y anómalas extendiéndose más allá de los límites normales e invadiendo partes adyacentes del cuerpo, propagándose a otros órganos. Éstas derivan de una única célula que en algún momento anterior ha experimentado una mutación que altera su programa normal de proliferación (1).

El cáncer oral es un serio problema que está aumentando en prácticamente todo el mundo. Es más común en pacientes de 50 años en adelante y en muchos países es más común en hombres que en mujeres, esto se debe a hábitos de riesgo o exposición al sol como parte algunas profesiones (4-6). Es una enfermedad multifactorial, en la que el tabaco y el alcohol juegan un papel muy importante, pero se puede prevenir evitando los factores de riesgo pertinentes, así como acudir a revisiones anuales al dentista (5).

El 90\% de los tumores malignos primarios son carcinomas orales de células escamosas, siendo más frecuente en borde lateral de la lengua (6) y su tratamiento dependerá del estadio en el que se encuentre la lesión $(4,7,8)$. El cese de hábitos como el tabaco y el alcohol, o cualquier factor de riesgo que pueda aumentar el riesgo de padecer cáncer oral, así como el diagnóstico temprano de las lesiones orales precancerosas y de cáncer de células escamosas en estadios iniciales pueden facilitar el tratamiento y ayudar a mejorar el pronóstico, reduciendo la mortalidad $(4,9,10)$.

\section{CARCINOGÉNESIS}

En el crecimiento normal de una célula se distingue la Interfase, en la que la célula crece hasta alcanzar un tamaño determinado para luego prepararse para su posterior división, y la División celular, en la que la célula se divide para formar dos células hijas. Este proceso recibe el nombre de Mitosis. La división celular está mediada por varios puntos de control que a su vez están regulados por proteínas reguladoras (11) (Fig. 1).

El desarrollo de cáncer en el ser humano es un proceso muy complejo que ocurre durante muchas décadas (1). La carcinogénesis es el mecanismo por el cual se desarrolla una neoplasia maligna (2). En los últimos años, se ha estudiado más en profundidad los mecanismos moleculares que se encuentran involucrados en el desarrollo del cáncer como la detección molecular del marcador antiapoptótico bcl2 , el marcador proapoptótico caspasa 3 y el marcador de proliferación celular Ki-67 que nos informarán sobre la situación más o menos grave del paciente respecto de su proceso canceroso. En este sentido, Bascones y cols. observan que las alteraciones en estas proteínas sumadas a los estados premalignos epiteliales crean estados celulares de hiperproliferación que favorecen la adquisición de errores oncogénicos confiriendo capacidad invasiva

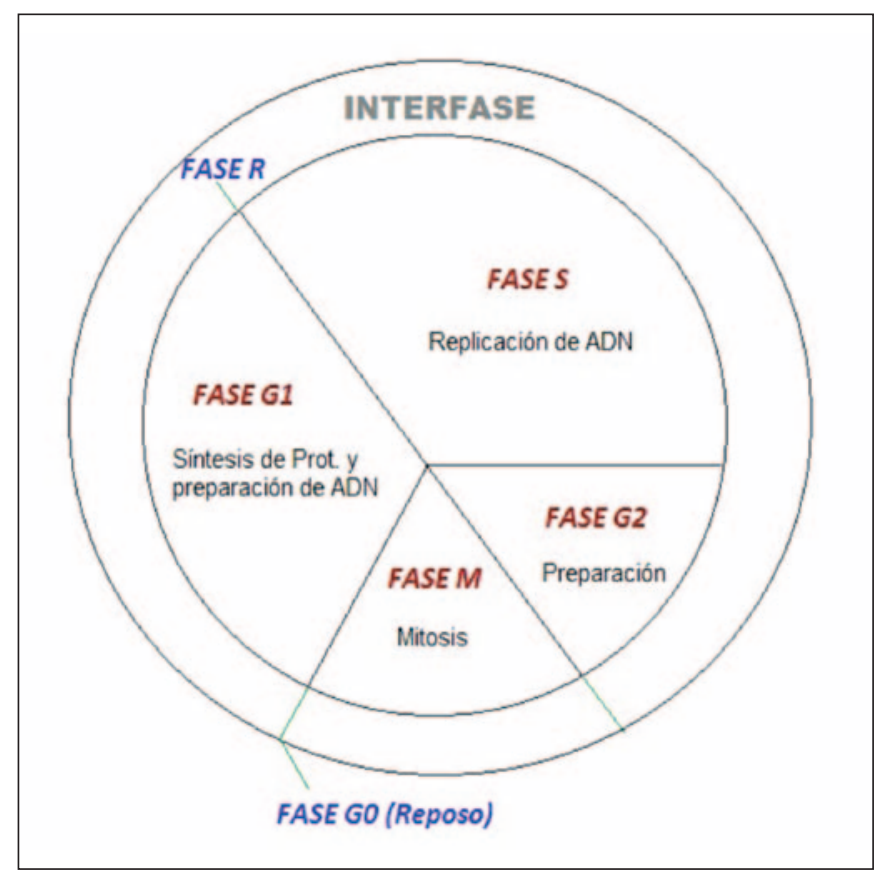

Fig. 1. Ciclo celular esquematizado en el que vemos la interfase, la célula crece hasta alcanzar un tamaño determinado para luego prepararse para la división celular representado por las fases G1, S, G2, M. Si hay algún fallo en la célula durante la división, ésta se destruirá en la fase R. La fase G0 dictamina el cese de la división celular y la célula pasará a un estado de reposo. 
(12). Las células están bajo la amenaza constante de varios factores citotóxicos y mutagénicos que dañan al ADN (13), éstos pueden ser endógenos o exógenos (14). Esta mutagenicidad se adquiere cuando los genes y proteínas que detectan y reparan el ADN son inactivados dando como resultado células mutagénicas y el posible sobrecrecimiento de los descendentes mutados, ya que además la apoptosis, proceso por el cuál se eliminan las células dañadas, está inactivo. La diferencia entre una célula normal y una neoplásica es la estimulación mitogénica, es decir, la proliferación celular normal depende de factores de crecimiento externos de su alrededor, mientras que las neoplásicas tienen una reducida dependencia debido a las actividades de los oncogenes (ras) que generan estimulación propia $(1,15)$. Numerosas observaciones nos indican que muchas, por no decir todas, las células tumorales comparten unos cambios fisiológicos, denominados capacidades adquiridas, suficientes para explicar el comportamiento maligno que caracteriza a las células cancerosas ya que incluyen habilidades como generar sus propias señales mitóticas, evitar apoptosis y capacidad para invadir y metastatizar $(1,12,15)$. La primera etapa del proceso de la carcinogénesis consta de tres etapas: Iniciación $(2,11,16)$, promoción y progresión (2). Hay varios genes encargados de la homeostasis celular, como los oncogenes, genes supresores de tumores y genes reparadores de ADN. Pero parece ser que en el desarrollo del cáncer, éstos se encuentran alterados $(1,2,15)$. Se ha visto que las alteraciones en la proteína P53, responsable de detener, temporalmente, el ciclo celular para reparar daños moleculares y bioquímicos en las células dañadas, así como de otras alteraciones que lleven a la inactivación de la maquinaria apoptótica, suelen llevar a una transformación maligna $(1,12,15)$.

Otro mecanismo para la formación tumoral se debe a la acción de la proteína telomerasa que activa una serie de mecanismos mediante los cuales se consigue que se produzca el mantenimiento de los telómeros, necesaria para que se produzca el tumor $(1,15)$.

\section{EPIDEMIOLOGÍA}

En España, el cáncer oral constituye un 2,6 \% de todos los tipos de tumores (Tabla 1) y su mortalidad es de 1,5 (Fig. 2) por cada 100.000 habitantes, cifra que se está acercando peligrosamente a la mortalidad del cáncer de cérvix uterino (1,9 por cada 100.000 habitantes) por lo que hay que empezar a darle más importancia. La incidencia del cáncer en 2013, en España, es de 6,7 por cada 100.000 habitantes (2,7 en mujeres y 11,0 en varones). El riesgo de padecer cáncer oral aumenta con la edad (en EE.UU, la media de edad para su diagnóstico es de 62 años durante 2000-2004) y la mayoría de los casos ocurren en personas de 50 años en adelante, aunque, en los estudios más recientes, se observan casos en adultos jóvenes $(4-6,17)$.

\section{FACTORES DE RIESGO}

La etiología del cáncer oral es multifactorial. El tabaco y el alcohol son dos de los factores más importantes, incluso usados conjuntamente, ya que aumentan de una manera drástica el riesgo de padecer cáncer oral y faríngeo, debido a su efecto sinérgico $(18,19)$. El consumo de nuez de betel o de areca, o hábitos tales como mascar tabaco y fumar invertido, son también factores muy importantes en la etiología del cáncer oral en países asiá-

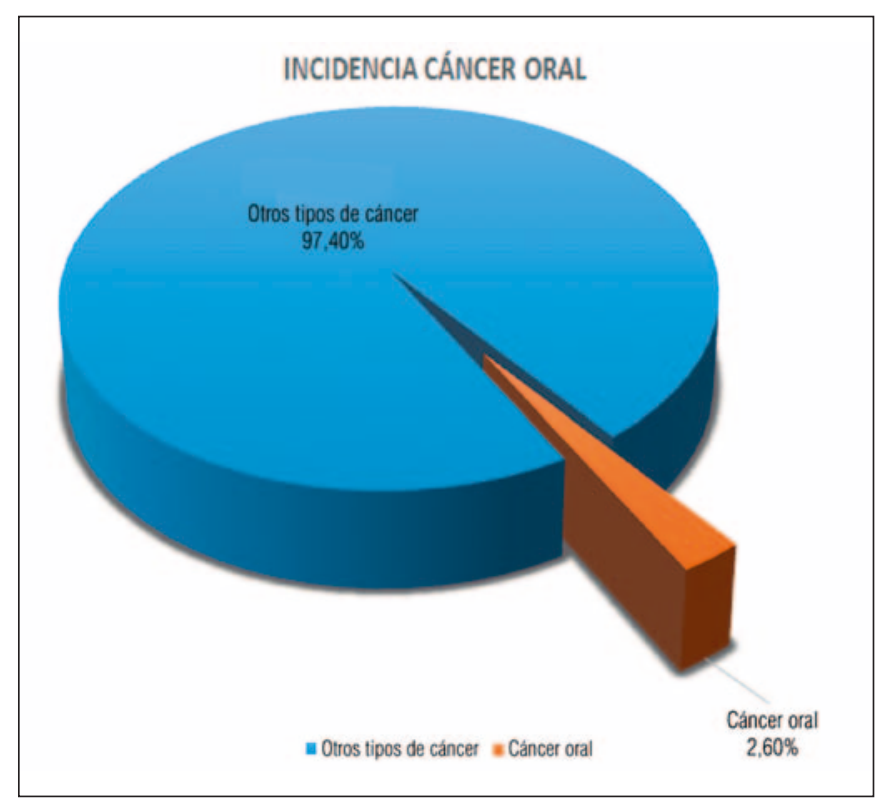

Fig 2. Incidencia de cáncer oral respecto a los demás tipos de tumores, en los cuales colorrectal (14,5\%), próstata (12,8\%), pulmón $(11,8 \%)$ y mama $(11,2 \%)$ son los más frecuentes. 
ticos o Suramérica (20). Algunos virus, como virus de ADN (hepatitis B y C), Epstein-Barr o VPH (genotipos 16 y 18), se engloban también dentro de la etiología. El aumento de edad, químicos en dieta, prótesis mal ajustadas, mala higiene oral, fármacos como el dietilestilbestrol o exposiciones a energías que producen daño directo a los genes como los rayos $\mathrm{X}$, son también factores a tener en cuenta $(4,15,21,22)$.

\section{ESTADO DE PRECÁNCER}

El término precáncer se refiere a aquellas presentaciones clínicas que pueden tener un potencial maligno para transformarse en cáncer. El precáncer oral tiene diversos aspectos clínicos que se engloban en los llamados trastornos potencialmente malignos, antiguamente conocidos como lesiones precancerosas o condiciones precancerosas, que pueden incrementar el riesgo de padecer cáncer oral $(4,9,23,24)$.

Dentro de éstas, las que normalmente preceden al cáncer oral son la eritroplasia y la leucoplasia. Según la OMS, la leucoplasia es "una mancha o placa blanca que no puede caracterizarse como otra entidad clínica ni patológica". Aparece después de los 40 años en adelante y está relacionada con el tabaco, alcohol o VPH (11 y 16), aunque también puede ser idiopática. Su localización varía según la localización geográfica o hábito de fumar; en países desarrollados el $90 \%$ de las lesiones aparecen en mucosa vestibular, comisuras, lengua o suelo de boca, mientras que en países como India, donde el hábito de fumar es distinto o se usa la nuez de betel, el $70 \%$ de las lesiones aparecen en paladar o mucosa labial inferior. Los valores de transformación maligna oscilan entre $0,13 \%-17,5 \%(10,25-28)$.

La eritroplasia se ve, con mayor frecuencia, en varones de mayor edad. Clínicamente, aparece como una mácula roja o en forma de placa aterciopelada. Histológicamente, se observan rasgos de que es carcinoma in situ y hasta un $85 \%$ más de displasias severas que en la leucoplasia, a pesar de que esta última es más frecuente, puede transformarse en maligna en un 3-33\% en diez años $(6,12,14-16)$.

\section{DIAGNÓSTICO}

Genéticamente, no hay ningún marcador fiable para detectar el cáncer oral por ahora. Se ha visto que durante una resección de cuello los marcadores CK13, CK19 y SCCA mRNA dieron positivo para cáncer. De todos estos, se vio que CK19 no es un marcador fiable, mientras que SCC mRNA parece ser un marcador muy fiable para el diagnóstico de las metástasis (29). Parece que un mecanismo esperanzador para la detección temprana en el cáncer y precáncer oral es el llamado mecanismo de senescencia, pero se necesitan más estudios para determinar cuales de estos marcadores pueden ser útiles en la carcinogénesis oral (30).

Clínicamente, en los estadios primarios las lesiones pueden ser asintomáticas y suelen ser pasadas por alto si el examen de la cavidad oral no es adecuado (7). Pero en otras ocasiones, o en estadios más avanzados nos podemos encontrar síntomas como sangrado bucal, pérdida de piezas dentarias, dificultad o dolor al deglutir, dificultad para llevar dentaduras, bultos en el cuello, úlcera que no cura, aparición de placas o lesiones de color blanco (leucoplasia) o rojo (eritroplasia), o una mezcla de lesión blanca y roja (eritroleucoplasia). Con el tiempo, estas lesiones se ulceran en su superficie y su crecimiento cambia evolucionando en una masa exofítica, o bien, en una lesión endofítica. A su vez, los signos que podemos observar son úlcera sin dolor, induración, parestesia de lengua o labio, crecimiento papilar y dificultad para abrir la boca debida a la disminución de la movilidad del tejido. Sospecharemos que una lesión pueda ser cáncer si persiste más de 3 semanas en boca $(4,19,31)$.

El cáncer oral puede afectar a cualquier parte de la cavidad oral pero su localización más frecuente suele ser en el borde lateral de la lengua y suelo de boca, aunque también puede afectar a otras estructuras como orofaringe, paladar blando, mucosa yugal o labio inferior. Se ha observado que cuando el paciente es fumador o ingiere alcohol, las lesiones se ven sobretodo en la zona anterior de lengua, suelo de boca, mucosa oral y alvéolos. Mientras que las lesiones por HPV aparecen más en las regiones posteriores de la cavidad oral como base de lengua, orofaringe, pilares amigdalinos y amígdalas (6). 
Lo primero será realizar una buena historia clínica, para encontrar posibles factores de riesgo que puedan producir que se desarrolle esta lesión a nivel oral, por ejemplo; si el paciente es fumador (el riesgo aumenta de cinco a nueve veces más en pacientes fumadores que en no fumadores) o sólo bebe (tiene treinta veces más posibilidad de desarrollar cáncer oral o faríngeo) o ambos (efecto sinérgico aumentando el riesgo cien veces más de lo normal), acompañada de una exhaustiva y buena exploración clínica, tanto extra como intraoral, la cuál requiere una adecuada iluminación, gasas $2 \times 2$, guantes de protección y espejos, gracias a los cuales, exploraremos todas las zonas de la cavidad oral (Tablas 1 y 2 ) $(4,18,19)$. En éste, se deberá examinar toda la mucosa oral de forma exhaustiva, además de incluir la palpación de los nódulos linfáticos cervicales. Prestando especial atención a las zonas de la boca que sean más propicias para el carcinoma; como son el labio, suelo de boca, bordes laterales de la lengua y mucosa yugal (7). El diagnóstico temprano depende de la astucia clínica o incluso del propio paciente cuando es capaz de identificar una lesión sospechosa mientras ésta se encuentra en un estadio temprano. Los estadios primarios pueden ser asintomáticos o causar pequeños cambios, por lo que se recomienda que los profesionales de la salud tengan un alto índice de sospecha y sepan identificar las lesiones orales precancerosas. Los pacientes que no acuden con frecuencia al dentista tienen más riesgo de diagnosticarles las lesiones en estadios muy avanzados. En el estudio llevado a cabo por Thomas Yu, se observó que hubo más retraso en el diagnóstico en las mujeres que en los hombres, pero se necesita investigar más el porqué de esta razón (32).

En muchas ocasiones, en caso de cualquier duda, los profesionales de la salud precisan de técnicas complementarias de apoyo al diagnóstico. La biopsia es un método que usamos como suplemento al diagnóstico, ésta se realizará cuando la lesión lleve más de 3 semanas en boca, lesiones que nos hagan sospechar de que pueden producir malignidad. Histológicamente, podemos observar que en el carcinoma hay una displasia extendida a lo largo del grosor del epitelio e invasión a través de la membrana (7).

Otro método diagnóstico es la técnica de screening, que analiza la presencia de enfermedad en una per-

\section{TABLA 1.- EXPLORACIÓN EXTRAORAL}

Cabeza y cuello, además de palpación bimanual de los nódulos linfáticos y glándulas salivales. Inspeccionar y palpar labio y zona del bermellón labial.

\section{TABLA 2.- EXPLORACIÓN INTRAORAL}

— Inspección y palpación de zona labial interna.

- Inspección y palpación de mucosa yugal.

- Inspección de encía vestibular y palatino/lingual, tanto adherida y libre, en maxilar superior e inferior.

- Inspección y exploración de la lengua:

- Para explorar el dorso de la lengua, le diremos al paciente que protruya la lengua.

- Para explorar la zona ventral, le diremos al paciente que lleve la punta de la lengua hacia el paladar.

- Para explorar los bordes laterales de la lengua, el paciente deberá protruir la lengua y nos ayudaremos de una gasa estéril tirando suavemente hacia los lados para inspeccionar y palpar la zona.

- Inspección y palpación de zona de paladar duro. Inspección paladar blando y orofaringe.

Tablas 1 y 2.- Pasos a seguir a la hora de realizar una buena exploración extra e intraoral. Para inspeccionar el paladar blando y la orofaringe nos ayudaremos de un espejo de exploración y, con él, bajaremos, suavemente, la lengua.

sona que no tiene síntomas. Los criterios para realizar esta prueba son tales como:

- La enfermedad debe ser un problema importante de salud.

- Debe haber un tratamiento aceptable para pacientes con esa enfermedad reconocida.

- Debe haber facilidades para el diagnóstico de la enfermedad.

- Debe haber un estadio sintomático temprano.

- El programa de screening debe ser costo-efectivo.

- La historia de la condición debe ser adecuadamente entendida, entre otras (6). 
Puesto que el cáncer cumple tres de los criterios expuestos, es una justificación para estas pruebas. Hoy en día las diferentes técnicas de screening abarcan desde un examen oral con luz incandescente, a pesar de que su uso sigue siendo controvertido, hasta la citología con cepillo, efectiva para aquellos pacientes que creemos que no volverán a futuras revisiones o que no acepten la derivación inmediata a un cirujano oral, o que presenten cinco áreas únicas problemáticas. Colorantes vitales como el azul de toluidina que tiñe los ácidos nucleicos o anormalidades del tejido son también ejemplos de técnicas que podrían utilizarse como screening, y aunque han sido usados durante muchos años presentan problemas como la falta de estudios controlados. Muchos estudios han valorado la eficacia de esta técnica como método útil, pero a día de hoy estos estudios no han podido decir con exactitud si el azul de toluidina puede ayudar a identificar y predecir el riesgo de progresión de lesiones que se escapan al ojo humano (6).

El Vizilite Plus o Microlux DL también sirven para descubrir anormalidades en el tejido tras enjuagarse el paciente con una solución de ácido acético al $1 \%$. Esta solución ayuda en el diagnóstico ya que elimina los restos del tejido para aumentar la visibilidad de éste. Tras el enjuague, observaremos el estado del tejido por medio de visualización directa aplicando luz blanco-azulada. Si la visualización del tejido nos da un color azul, nos indica que éste es normal, si por el contrario lo observamos de color blanco, nos indicará anormalidades en el tejido. Velscope es un dispositivo móvil que permite también la visualización directa de la mucosa oral mediante una luz azul de 400-600 nm, en éste, la mucosa oral normal se tornará de un color verde mientras que si observamos un color negro significará que hay anormalidades en la mucosa (6).

\section{PREVENCIÓN}

Tres de cada cuatro cánceres orales pueden ser prevenidos eliminando el consumo de tabaco y alcohol, reduciendo al mismo tiempo el riesgo de tumores secundarios. También reducir el hábito de betel en Asia ayuda a la prevención. Se ha visto que el consumo de fruta y vegetales reduce el riesgo de padecer cáncer oral, lo que hace suponer que una dieta de- ficiente en antioxidantes es un factor que predispone al desarrollo de cáncer y al estado de precáncer $(11,14,16)$.

Los exámenes anuales por dentistas deberían estar centrados, sobretodo en aquellos pacientes con más de 60 años de edad con factores de riesgo como lesiones orales premalignas o que consuman alcohol o tabaco. Pero esto no significa que el resto de la población no deba de ser explorada de forma exhaustiva por su dentista todos los años. Aunque las lesiones precancerosas orales son relativamente infrecuentes, su relativa importancia de transformación maligna nos pueden ayudar a encontrar estrategias para la prevención, en las poblaciones de alto riesgo. La educación pública también es algo que se necesita como prevención. Thomas Yu, aconseja que para aumentar la supervivencia necesitamos programas de educación pública para fomentar que los pacientes eviten comportamientos de riesgo, además de exámenes con métodos de screening $(11,16,32)$.

\section{ANGIOGÉNESIS Y METÁSTASIS}

La angiogénesis es una de las características esenciales en la formación de tumores y es también muy importante en el desarrollo, reproducción y curación de éstos. En las células normales los vasos sanguíneos se encuentran en un estado quiescente y las células secretan bajos niveles de inducción y altos niveles de inhibición. Mientras que en las células malignas es al contrario. Muchas moléculas que se observan en el cáncer de cabeza y cuello, son capaces de inducir angiogénesis y están directamente producidas por los queratinocitos. Muchos estudios explican que la interleuquina 8 (Il-8) tiene también un mayor efecto angiogénico. Se piensa que el contenido del estroma está indirectamente relacionado con la inducción de la angiogénesis (33-36).

La metástasis es, aparte de un proceso selectivo ("Seed and Soil", 2003) (37), uno de los aspectos más temidos del cáncer ya que es la propagación de las células tumorales desde donde se encuentra la neoplasia primaria a los distintos órganos del cuerpo, además de su crecimiento implacable que además necesita el desarrollo de un suministro de vasos 
sanguíneos, permitiendo que las células pasen al torrente sanguíneo (intravasación) y lleguen a un órgano distinto en donde iniciarán y seguirán el crecimiento de nuevas metástasis. Se ha observado que el número de metástasis que se desarrollan es directamente proporcional al número de células tumorales inyectadas intravascularmente, así como ser un proceso que depende de factores mecánicos y el secuestro capilar. Los tumores metastáticos de la cavidad oral son poco comunes, lo cuál supone el $1 \%$ de las neoplasias malignas orales. Se ha visto que la frecuencia de micrometástasis es mucho mayor cuando se trata de cáncer de cabeza y cuello (33-36).

\section{ESTADIAJE TNM}

El sistema de estadiaje TNM fue propuesto en 1941 por Pierre Denoix. Es un sistema anatómico a través del cuál se describe la extensión, a nivel anatómico, del tumor primario así como si ha afectado a nivel de los nódulos linfáticos y si ha producido metástasis a distancia. Hoy en día, se utiliza el sistema TNM desarrollado por la AJCC (American Joint Comittee on Cancer) y en colaboración con la UICC (Unión Internacional Contra el Cáncer), actualizado en varias ocasiones hasta la última publicada en 2003 (Tabla 3). Es muy importante el uso de este sistema de estadiaje para establecer un diagnóstico adecuado y un adecuado tratamiento $(4,38-40)$.

\section{TRATAMIENTO}

Las opciones de tratamiento para los carcinomas orales, son variables y dependen de varios factores como del tamaño y localización del tumor primario, del estado de los ganglios linfáticos, presencia o ausencia de metástasis a distancia, o incluso, de los deseos del propio paciente y de la capacidad que tenga para tolerar el tratamiento. Cuando un paciente presenta un carcinoma oral la supervivencia en los estadios tempranos es del $80 \%$ mientras que en los avanzados es de 35\%. Para el tratamiento de este tipo de cáncer se debe intentar preservar la máxima estructura sana posible. Tanto la cirugía como la radioterapia, siguen siendo el gold standard para el tratamiento de los tumores de labio y cavidad oral,
TABLA 3.- ESTADIAJE TNM DE TUMORES DE CAVIDAD ORAL TOMADA DE "PATEL SG, SHAH JP. TNM STAGING OF CANCERS OF HEAD AND NECK: STRIVING FOR UNIFORMITY AMONG DIVERSITY. CA CANCER J CLIN 2005;55:242-58”

T (Descripción del tamaño del tumor primario)

Tx El tumor primario no puede ser evaluado.

T0 No hay evidencia de tumor primario.

Tis Carcinoma in situ.

T1 Tumor de $2 \mathrm{~cm}$ de tamaño o menor.

T2 Tumor entre $2-4 \mathrm{~cm}$.

T3 Tumor mayor a $4 \mathrm{~cm}$.

T4 Tumor invade otras estructuras como; el hueso cortical, seno maxilar, piel, músculo pterigoideo (T4a, T4b).

N (estado de los ganglios linfáticos regionales)

Nx Los ganglios linfáticos regionales no pueden ser evaluados.

NO No hay evidencia de tumor primario.

N1 Metástasis a nivel de un solo nódulo linfático ipsilateral de $3 \mathrm{~cm}$ o menor.

N2 Metástasis a nivel de un solo nódulo linfático ipsilateral entre 3-6 cm de tamaño (2a), metástasis en múltiples nódulos linfáticos ipsilaterales no mayores de $6 \mathrm{~cm}(2 \mathrm{~b})$ o metástasis a nivel de nódulos linfáticos bilaterales no mayor de $6 \mathrm{~cm} \mathrm{(2a)}$.

N3 Metástasis en un nódulo linfático mayor de $6 \mathrm{~cm}$.

M (metástasis a distancia)

M0 No hay metástasis a distancia.

M1 Hay metástasis a distancia.

ya sea usados por separado, o en combinación con quimioterapia en estadios avanzados $(4,7,8)$.

La radioterapia juega un papel muy importante en el tratamiento del cáncer de cabeza y cuello, tal es así que los nuevos casos de cáncer van a necesitar la radioterapia como tratamiento inicial, como coadyu- 
vante a la cirugía, o combinado con quimioterapia. La dosis de la radioterapia van a depender de la localización y tipo de tumor, además de si la radiación va a ser usada de forma única o en combinación con otras modalidades. Normalmente, las dosis de radiación en un paciente con cáncer de cabeza y cuello llegan hasta 50-70 Gy, en un periodo de 5 a 7 semanas, una vez al día, 5 días a la semana. Una de las nuevas técnicas es añadir cisplatino en el tratamiento de la radioterapia para ver si puede mejorar los resultados en el tratamiento, no obstante, en el estudio presentado por S. Cooper, y tras analizar a 459 pacientes de los cuales 231 fueron asignados al azar para recibir radioterapia y 228 pacientes radioterapia además de cisplatino, llegó a la conclusión de que la radioterapia y la quimioterapia mejoran significativamente el control locorregional, además de aumentar la supervivencia. Sin embargo, el tratamiento combinado de radioterapia y cisplatino no hace más que aumentar los efectos adversos haciendo que 4 pacientes del presente estudio fallecieran por ello. En muchos estudios que comparan tanto la cirugía como la radioterapia, se ha observado que la supervivencia depende del estadio en el que se encuentra la enfermedad cuando se diagnostica. Pero la radioterapia tiene también complicaciones graves que afectan a estructuras orales como glándulas salivales, hueso, dentición y mucosa oral, entre otros, provocando en el paciente consecuencias clínicas como mucositis, xerostomía, osteorradionecrosis y caries por radiación (41-48). Todas estas consecuencias tienen un gran impacto en la calidad de vida del paciente por ello hay un nuevo método terapéutico como es el de la braquiterapia, tipo de radiación interna local, que se encuentra en contacto con la masa tumoral a través de unos adaptadores, como agujas o tubos, que son cómodos y no producen daño. Es una técnica poco lesiva ya que ofrece una radiación menor que la radioterapia externa, además de evitar que la radiación no llegue a los tejidos vecinos sanos gracias a un protector de acrílico de $2 \mathrm{~mm}$ de plomo. Pero no en todos los casos se puede usar esta terapia, y es que cuando el hueso está afectado, o haya pérdida importante de tejido o necesite una gran escisión quirúrgica, está totalmente contraindicado utilizar esta técnica. Con el uso de esta técnica hay que considerar que en algunos casos puede producir una pequeña mucosi- tis en la zona donde aplicamos la radiación, pero este inconveniente suele desaparecer entre 1-1,5 meses $(8,10,40,49)$.

El tipo de cirugía dependerá de si los nódulos cervicales están afectados o no. Si no lo están, nos bastará con extirpar toda la lesión con márgenes de seguridad, además de los ganglios cervicales. Si por el contrario, éstos si están afectados procederemos a realizar una cirugía radical estándar, extirpando el músculo ECM, milohioideo, nervio IX, glándula submaxilar, venas yugulares, sistema linfático cervical y polo inferior de parótida e incluso estructuras óseas vecinas si el tumor es muy grande. El tratamiento para el cáncer oral compromete sobre todo la función del habla, llevando a una reducida inteligibilidad. Cuanto más grande es el tumor, más comprometido estará el habla $(8,49)$.

Para el tratamiento de las lesiones premalignas, por lo general, se recomienda que aquellas leucoplasias que muestren una displasia de tipo leve, pueden revertir si el factor de irritación se elimina (ejemplo el tabaco), mientras que aquellas leucoplasias que presenten displasia de tipo moderada o severa, sean extirpadas siempre que sea posible. Aparte de la cirugía, también podemos intentar eliminar estas lesiones con las terapias no invasivas, entre ellos encontramos los carotenoides, licopenos, los cuales necesitan más estudios, o ácido retinoico como la terapia con 13cRA que es muy efectiva, pero produce un incremento de los efectos adversos tanto durante el tratamiento así como cuando dejamos de usar esta terapia. A pesar de ser fáciles de aplicar para el paciente y ser de bajo coste, hay que tener muy en cuenta la recurrencia que tiene la leucoplasia una vez eliminada $(4,50,51)$.

Se ha observado que la talidomida tiene efecto anticancerígeno, usada en combinación con otros medicamentos destinados a tratar el cáncer, como Paclitaxel o Cisplatin, pero estos datos han sido obtenidos in vitro y no se pueden extrapolar a estudios humanos, por lo que se debería investigar más su potencial anticancerígeno y realizar más estudios sobre su verdadero resultado en el cáncer (52).

Otra opción de tratamiento no invasivo para los tumores de cabeza y cuello, así como para las lesiones 
premalignas, es la llamada terapia Fotodinámica (TFD), que en comparación con la radioterapia y la quimioterapia, es más segura ya que las sustancias que se utilizan tienen preferencia, en acumulación, por las células tumorales preservando los tejidos sanos de alrededor, disminuyendo los efectos secundarios y el coste. Esta terapia se usa con frecuencia en EEUU, Canadá y algunos países de Europa pero en otros, como Brasil, está aún en experimentación. Según el metaanálisis llevado a cabo por Biel, la TFD presenta unos resultados muy prometedores, pero aún se necesitan más estudios para corroborar esta efectividad $(53,54)$.

\section{PRONÓSTICO}

Los pacientes de cáncer oral tienen muchas posibilidades de padecer otro cáncer, que puede darse de 5 a 10 años desde el primero. En muchos países, los valores de supervivencia para el cáncer de lengua, cavidad oral y orofaringe son $50 \%$ mientras que, para el cáncer de labio, la supervivencia es del $90 \%$. La supervivencia está directamente relacionada con el estadio en el que se diagnostique el tumor, así como a la prevención y al diagnóstico temprano. Hay que tener en cuenta que la presencia o ausencia de metástasis en los nódulos linfáticos cervicales es el factor pronóstico más importante para los pacientes con carcinoma oral de células escamosas $(4,5,19,35)$.

Pacientes que han padecido cáncer oral, tienen un $15 \%$ de posibilidades de padecer un segundo tumor primario, por lo que es muy importante que en cada revisión se les realice un completo examen de cabeza y cuello, incluyendo, la laringe. El control de los factores de riesgo tras ser tratados de cáncer oral, es de vital importancia, ya que se ha visto que aquellos pacientes que continúan fumando después del tratamiento, tienen de dos a seis veces más posibilidades de desarrollar un segundo tumor en el tracto aerodigestivo, que aquellos que cesan el hábito (4).

Muchos pacientes con cáncer oral que han sido tratados con éxito deben de hacer frente, tras el tratamiento, a las devastadoras consecuencias. Como ya se ha comentado previamente, el tratamiento en sí del cáncer oral es bastante agresivo ya que se someten a tratamientos que pueden afectar a la apariencia y función. Los problemas en la calidad de vida son especialmente importantes en este grupo de pacientes, ya que pueden desembocar en una depresión o en un déficit nutricional. Es por ello, que estos pacientes deben ser controlados de una manera multidisciplinar y coordinada por un equipo formado por profesionales de la salud como cirujano maxilofacial, oncólogo, dentista general, patólogo general, prostodoncista maxilofacial, enfermera, higienista dental, nutricionista, logopeda, entre otros, para ayudarle y mejorar su situación $(4,5)$.

\section{DISCUSIÓN}

En el cáncer, casi todas las estirpes celulares se encuentran afectadas. Éstas han sido estudiadas desde hace muchos años, progresando en el tiempo. La etiología del cáncer oral es multifactorial y una de sus particularidades es que se aumentan las probabilidades de padecerlo con la edad, observándose en pacientes de 50 años en adelante y más en varones. Otros factores etiológicos que aumentan el riesgo son el tabaco, el alcohol, lesiones premalignas o virus, tales como el papiloma humano, entre otros.

El carcinoma oral de células escamosas es el tumor maligno más frecuente que nos podemos encontrar en la cavidad oral, con una frecuencia del $90 \%$. Éste suele presentarse, clínicamente, como una lesión blanca (leucoplasia), lesión roja (eritroplasia) o una mezcla de lesión roja y blanca que con el tiempo puede producir la ulceración de la superficie mucosa. Su evolución puede ser de crecimiento de tipo exofítico o endofítico caracterizado por una superficie ulcerada. La localización más frecuente de este tipo de tumores es borde lateral y superficie ventral de la lengua, seguido de suelo de boca. Por ahora, el único método eficaz contra el cáncer oral es la prevención, por parte del paciente, evitando los factores de riesgo pertinentes o incluso de identificarse cualquier lesión sospechosa que le pueda aparecer en boca o labio y un diagnóstico precoz de la enfermedad, por parte del odontólogo o el médico de Atención Primaria. Una correcta Historia Clínica 
junto con una exploración exhaustiva extraoral e intraoral, teniendo especial cuidado en las zonas más frecuentes de aparición de lesiones cancerosas o lesiones premalignas, ayudándonos de pruebas complementarias como screening o biopsiando toda aquella lesión que persista más de dos o tres semanas en boca para descartar la posibilidad que sea un cáncer.

La extensión y la localización del tumor, el estado de los ganglios linfáticos, la presencia o ausencia de metástasis a distancia, o incluso, de los deseos del propio paciente y la capacidad que tenga para tolerar el tratamiento, son factores que condicionan las opciones a la hora de seleccionar el mejor tratamiento. Para ello usaremos el sistema de estadiaje TNM, que ayudará a la hora de establecer un correcto diagnóstico y, a su vez, el tratamiento adecuado. Por ahora, el gold standard para el cáncer oral es la cirugía y la radioterapia, pero si la lesión está muy avanzada, el tratamiento más efectivo, por ahora, es la combinación de radioterapia con quimioterapia, además de la cirugía. El problema es que este tipo de tratamientos disminuyen bastante la calidad de vida del paciente, por lo que se debería de estudiar algún otro método menos agresivo para combatir esta enfermedad, aunque por ahora el diagnóstico precoz es el mejor método para ello.

Por lo tanto, el riesgo de padecer cáncer oral aumenta con la edad, en especial en pacientes de 50 años en adelante. Factores de riesgo tales como el tabaco, el alcohol, lesiones potencialmente malignas, factores genéticos y algunos tipos de virus, aumentan las probabilidades de padecerlo. Su diagnóstico deberá ser precoz ya que así se podrá mejorar el pronóstico de estos pacientes, por lo que se recomienda a todos los odontólogos, así como a los médicos de Atención Primaria, que realicen una exhaustiva exploración de la mucosa oral, teniendo en cuenta los factores de riesgo y se deberá biopsiar cualquier lesión que persista más de dos semanas en boca.

El riesgo de padecer cáncer oral aumenta con la edad, en especial en pacientes de 50 años en adelante. Factores de riesgo tales como el tabaco, el alcohol, lesiones potencialmente malignas, factores genéticos y algunos tipos de virus, aumentan las probabilidades de padecerlo. Su diagnóstico deberá ser precoz ya que así se podrá mejorar el pronóstico de estos pacientes, por lo que se recomienda a todos los odontólogos, así como a los médicos de Atención Primaria, que realicen una exhaustiva exploración de la mucosa oral, teniendo en cuenta los factores de riesgo y se deberá biopsiar cualquier lesión que persista más de dos semanas en boca.

\section{BIBLIOGRAFÍA}

1. Hahn WC, Weinberg RA. Rules for making human tumor cells. N England J Med 2002;347:1593603.

2. García-García V, González-Moles MA, Bascones Martínez A. Bases moleculares del cáncer oral. Revisión bibliográfica. Av Odontoestomatol 2005; 21(6):287-95.

3. Díez-Pérez R, Campo-Trapero J, Cano Sánchez J, López-Durán M, González Moles MA, Bascones-Ilundain $\mathrm{J}$ et al. Methylation in oral cancer and precancerous lesions (Review). Oncol Rep 2011;25:1203-9.

4. Neville BW, Day TA. Oral cancer and precancerous lesions. CA Cancer J Clin 2002;52: 195-215.

5. Warnakulasuriya S. Review Global Epidemiology of Oral and Oropharyngeal Cancer. Oral Oncol 2004;25:309-16.

6. Lingen MW, Kalmarr JR, Karrison T, Serbit PM. Critical evaluation of diagnostic aids for the detection of oral cancer. Oral Oncol 2008;44(1): 10-22.

7. Cooper JS, Pajak TF, Forstiere AA, Jacobs J, Campbell B. Postoperative concurrent radiotherapy and chemotherapy for high risk squamous cell carcinoma of the head and neck. N Engl J Med 2004;350(19):1937-44.

8. Vissink A, Jansma J, Spijkernet FKL, Burlage FR, Coppes RP. Oral sequeale of head and neck 
radiotherapy. Crit Rev Oral Bio Med 2003;14(3): 199-212.

9. Aguas SC, Ianfranchi HE. Lesiones premalignas o cancerizables de la cavidad oral. Rev Fac Odont (UBA) 2004;19(47):21-30.

10. Bermejo ME, Martínez AB. Leucoplasia oral: Conceptos actuales/Oral Leukoplakia: Current considerations. Av Odontoestomatol 2009;25(2): 83-97.

11. Morange M. What history tells us XXVIII. What is really new in the current evolutionary theory of cancer. J Biosci 2012;37(4):609-12.

12. Bascones-Martínez A, Rodríguez-Gutiérrez C, Rodríguez-Gómez E, Gil-Montoya JA, GómezFont R, González-Moles MA. Evaluation of P53, Caspase-3, Bcl-2, and Ki-67 markers in oral squamous cell carcinoma and premalignant epithelium in a sample from Álava province (Spain). Med Oral Patol Oral Cir Bucal 2013;18 (6):e846-50.

13. Norbury CJ, Hickson ID. Cellular responses to DNA damage. Annu Rev Pharmacol Toxicol 2001;41:367-401.

14. Jané-Salas E, Chimenos-Küstner E, LópezLópez J, Roselló-Llabrés X. Importancia de la dieta en el cáncer oral. Med Oral 2003;8:2608.

15. Katayama H, Brinkley WR, Sen S. The Aurora Kinases: role in cell transformation and tumorogenesis. Cancer Metastasis Rev 2003;22 (4):451-64.

16. Cairns J. Mutation selection and the natural history of cancer. Nature 1975; 255:197-200.

17. SEOM. La situación del cáncer en España/ El cáncer en España. Madrid (España): SEOM 2013.

18. Pelucchi C, Gallus S, Garavello W, Bosetti C, La Vecchia C. Cancer risk associated with alcohol and tobacco use: focus on upper aero-digestive tract and liver. Alcohol Research \& Health 2007; 29(3):193-8.

19. Osuna T, Hopkins S. Oral cancer diagnostic Technologies. CDHA Journal 2012;24(1):12-8.

20. Zain RB, Ikeda N, Gupta PC, Warnakulasuriya S, Werner Van Wyk C, Shrestha P et al. Oral mucosal lesions associated with betel liquid, areca nut and tobacco chewing habits. J Oral Pathol Med 1999;28(1):1-4.

21. Loyha K, Vatanasapt P, Promthet S, Parkin DM. Risk factors for oral cancer in Northeast Thailand. Asian Pac J Cancer Prev 2012;13:5087-90.

22. Quintana Díaz JC, Fernández Fregio MJ, Laborde Ramos R. Factores de riesgo de lesiones premalignas y malignas bucales. Rev Cubana Estomatol 2004;41(3):10-5.

23. Warnakulasuriya S, Johanson NW, Van der Waal I. Nomenclature and classification of potentially malignant disorders of the oral mucosa. J Oral Pathol Med 2007;36(10):575-80.

24. Warnakulasuriya S. Oral epidemiology of oral and oropharyngeal cancer. Oral Oncol 2009;45:30916.

25. Banoczy J. Oral leukoplakia and other white lesions of the oral mucosa related to dermatological disorders. Journal Cutan Pathol 1983; 10:238-56.

26. Van der Waal I. Potentially malignant disorders of the oral and oropharyngeal mucosa; terminology, classification and present concepts of management. Oral Oncol 2008;45:317-23.

27. Schepman KP, Bezemer PD, Van der Meij, Smeele LE, Van der Waal I. Tobacco usage in relation to the anatomical site of oral leukoplakia. Oral Dis 2001;7:25-7.

28. Mehta FS, Pindborg JJ, Gupta PC, Daftary DK. Epidemiologic and histologic study of oral cancer and leukoplakia among 50,915 villagers in india. Cancer 1969;24:832-49. 
29. Beckman RA, Loeb LA. Review genetic instability in cancer: theory and experiment. Semin Cancer Biol 2005; 15:423-35.

30. Campo-Trapero J, Cano-Sánchez J, López-Durán $M$, Palacios-Sánchez $B$, Sánchez-Gutiérrez JJ, Bascones-Martínez A. Marcadores de senescencia celular en cáncer y precáncer oral. Av Odontoestomatol 2008;24(1):69-80.

31. Rocha A. Oral cancer: The role of the dentist in early diagnosis and control/Cáncer oral: El papel del odontólogo en la detección temprana y control. Rev Fac Odontol Univ Antioq 2009;21:112-21.

32. Yu T, Wood RE, Tenenbaum HC. Delays in diagnosis of head and neck cancers. J Can Dent Assoc 2008;74(1):61.

33. Chambers AF, Groom AC, MacDonald IC. Dissemination and growth of cancer cells in metastatic sites. Nat Rev Cancer 2002;2:563-72.

34. Hasina R, Lingen MW. Angiogenesis in oral cancer. J Dent Educ 2001;65(11):1282-90.

35. Van Den Brekel BMW, Stel HV, Van Der Valk P, Van Der Waal I, Meyer CJLM, Snow GB. Micrometastases from Squamous cell carcinoma in neck dissections specimens. Eur Arch Otorhinolaryngol 1992;249:349-53.

36. Chen YK, Wang WC, Chen JY, Lin LM. Oral soft tissue metastases. J Clin Family Dent 2009;3 (4):25-30.

37. Fidler IJ. The patogenesis of cancer metastasis: The "seed and soil" hypothesis revisited. Nat Rev Cancer 2003;3:1-6.

38. Denoix PF. Tumor, Node and Metastasis (TNM). Bull Inst Nat Hyg 1944; 1:1-69.

39. Patel SG, Shah JP. TNM staging of Cancers of Head and Neck: Striving for Uniformity Among Diversity. CA Cancer J Clin 2005; 55: 242-58.

40. Casino AR, Toledan IP, Jorge JF, Díez JMO, Llobet CC, Prat MA. Brachytherapy in lip cancer. Med Oral Patol Oral Cir Bucal 2006;11:E223-9.
41. Vaishali MR, Roopashri G, Maria Priscilla David, Indira AP. Trismus. Indian J Dent Adv 2010;2 (3):303-8.

42. Gómez AS, Del Castillo CAL, Fuentes LE, Caballero GQ. Consecuencias de la radioterapia en las afecciones de Cabeza y Cuello. Archivo Médico Camaguey 1998;2(1):1-5.

43. Kiprian D, Kamecki A, Rolski W, Michalsk W. The impact of xerostomy on the quality of life in irradiated patients with head and neck cancer. $J$ Oncol 2009;1:E22-6.

44. Jiménez JS, Herrera CAR, Blanco FA, Arévalo REA, Martínez MM. Manejo Terapéutico de la xerostomía en Atención Primaria. Medicina de familia. 2004;1:42-9.

45. Milena Molma Giraldo S, René Estupiñán Guzmán J. Mucositis Oral en el paciente con terapia antineoplásica. Medicina UPB 2010; 29(2):13543.

46. Silverman JRS. Diagnosis and Management of Oral Mucositis. J Support Oncol 2007;5:13-21.

47. Dangi YS, Soni ML, Namdeo KP. Oral Candidiasis: A review. J Pharm Pharm Sci 2010;4:3641.

48. Gevorgyan A, Wong K, Poon I, Blanas N, J Enepekides D, M Higgins K. Osteoradionecrosis of the mandible: a case series at a single institution. Otolaryngology-Head and Neck Surgery 2013;42:1-7.

49. Schuster M, Stelzle F. Outcome measurements after oral cancer treatment: Speech and speech related aspects- an overview. Oral Maxillofac Surg 2012;16:291-8.

50. Singh SK, Gupta A, Sahu R. Non-surgical Management of Oral Leukoplakia. J Dent Scien 2013;2(2):39-47.

51. Ribeiro AS, Salles PR, Da Silva TA, Mesquita RA. A review of the nonsurgical treatment of oral leukoplakia. Int J Dent 2010;1:1-10. 
52. Jin X, Lu S, Xing X, Wang L, Mu D, He M, Huang $\mathrm{H}$, Zeng X, Chen $\mathrm{Q}$. Thalidomide: features and potential significance in oral precancerous conditions and oral cancer. J Oral Pathol Med 2013;42:355-62.

53. Bicalho L, Joao L, Pereira L, Santos MF, Azevedo R. Photodynamic therapy, a new approach in the treatment of oral cancer: [review]/Terapia fotodinámica, un nuevo acercamiento en el tratamiento del cáncer oral [revisión]. Rev Univ Ind Santander Salud 2010;42 (2):162-74.
54. Biel MA. Photodynamic therapy treatment of early oral and laryngeal cancers. Photochem Photobiol 2007;83:1063-8.

\section{CORRESPONDENCIA}

Ma Cristina Mateo-Sidrón Antón

Paseo Pintor Rosales, 76

28008 Madrid.

Correo electrónico: cristina.msa@outlook.e 